Original Article

Journal of Epilepsy Research pISSN 2233-6249 / elSSN 2233-6257

Received October 9, 2015

Accepted November 14, 2015

Corresponding author: Dong Wook Kim

Department of Neurology, Konkuk

University School of Medicine, 120-1

Neungdong-ro, Gwangjin-gu, Seoul

05030, Korea

Tel. $+82-2-2030-7562$

Fax. +82-2-2030-7469

E-mail; drdongwkim@kuh.ac.kr

\title{
Safety and Efficacy of Zonisamide in Patients with Epilepsy: A Post-Marketing Surveillance Study
}

\author{
Hye Jin Lee', Jeong Min Son ${ }^{2}$, Jihee Mun², Dong Wook Kim ${ }^{1}$ \\ ${ }^{I}$ Department of Neurology, Konkuk University School of Medicine, and ${ }^{2}$ Medical Department, Eisai Korea Inc., \\ Seoul, Korea
}

Background and Purpose: Zonisamide (ZNS) is one of new antiepileptic drug, which is known to inhibit seizure through multiple mechanisms of action. In Korea, ZNS was approved as an antiepileptic drug in 1992 and has been used for epilepsy patients with partial and generalized seizures. The objective of this study was to investigate the efficacy and tolerability of ZNS in patients with epilepsy and to identify the incidence of adverse events in real clinical setting.

Methods: This study was carried out in patients who received ZNS for epilepsy. Patients who were observed for at least 12 weeks after treatment with ZNS were included as evaluable subjects. Information regarding the status and type of adverse events occurring during the course of treatment with ZNS was obtained regardless of causal relationship to ZNS and efficacy was assessed by the study physicians and patients at 12 weeks post dose of ZNS.

Results: A total of 1,948 patients were included in the study, and ZNS efficacy was evaluated in 1,744 patients. ZNS was used as a monotherapy in 1,095 patients and as an adjunctive drug in 853 patients. Of the total patients, 1,345 (69.1\%) patients had partial seizure, 563 patients had generalized seizure, and 40 patients were undetermined. Adverse events were reported in 65 patients (3.34\%) including 1 case of Stevens-Johnson syndrome, but no incidence of serious unexpected adverse drug reactions were reported. 755 patients $(43.29 \%)$ became seizure free with ZNS treatment, and additional 322 patients (18.41\%) experienced marked improvement with ZNS treatment.

Conclusions: Our study shows the safety and tolerability of ZNS treatment in patients with epilepsy in real clinical setting. In addition, ZNS was found to be an effective option as a monotherapy or in patients with generalized seizure. (2015;5:89-95)

Key words: Epilepsy, Zonisamide, Post-marketing surveillance

\section{Introduction}

Zonisamide (ZNS) is one of the second generation antiepileptic drugs (AEDs). It is chemically unrelated to other AEDs and has a broad spectrum of action mechanisms including inhibition of $\mathrm{Na}^{+}$ channels, reduction of $\mathrm{T}$-type $\mathrm{Ca}^{2+}$ currents, reduction in glutamate-mediated synaptic excitation, and enhanced inhibitory effects mediated by gamma-aminobutyric acid. This drug was approved by the US Food and Drug Administration in 2000 and by the European Union in 2005. In Korea and Japan, ZNS has been used for more than 20 years for the treatment of partial and generalized seizures as monotherapy or adjunctive therapy, and even for use in pediatric patients. $^{1-3}$
ZNS shows a relatively favorable tolerability profile and maintenance rate among the AEDs ${ }^{4}$ and its efficacy and safety have been demonstrated in a number of study reports published in other countries. ${ }^{5-7}$ In Korea, although the drug has been widely used for the treatment of epilepsy since its approval, its post-marketing surveillance has not been carried out systematically. In addition, the previous clinical studies were carried out under limited conditions with a small sample size of no more than 200 subjects, ${ }^{1,8}$ suggesting the lack of sufficient safety information collected in the clinical setting.

In this context, the primary objective of this study was to investigate and collect safety information on ZNS in the clinical setting, including expected and unexpected adverse events (AEs). The secondary objective of this study was to evaluate the tolerability and effi- 
cacy of ZNS treatment in patients with epilepsy.

\section{Methods}

\section{Patients}

This study was carried out in patients treated with ZNS for the indication of epilepsy including partial seizure, generalized seizure including tonic-clonic seizure, tonic seizure, and atypical absence, and unclassified seizure between May 2011 and August 2013. Patients were included upon providing verbal consent to access to and use of their private and medical data. Patients were excluded from analysis if they had past or current hypersensitivity reactions to the study drug or if they had several hereditary problems, such as galactose intolerance, Lapp lactase deficiency, or glucose-galactose malabsorption. The investigation and collection of clinical data were conducted in institutions that concluded a written contract and initiated following the review by the Institutional Review Board of each institution, as necessary.

Patients who were observed for at least 12 weeks after treatment with ZNS, who had received at least one dose of ZNS, and for whom safety information was obtained through post-dose visits were acceptable as evaluable subjects.

\section{Sample size estimation}

This study aimed to assess the safety of ZNS by collecting information on unexpected AEs (including renal stone as a serious AE) in epilepsy patients. The sample size was set in a manner to be sufficient to detect uncommon and serious AEs in patients with epilepsy upon administration of ZNS. The sample size was calculated by using the incidence rate of AEs of renal stone $(\lambda)$ and the estimation was based on the literature. Based on previous studies on monotherapy, the incidence rate of renal stone was predicted to be $0.13 \%,{ }^{7}$ and the sample size was calculated using the following formula to identify one incidence (a) at a $95 \%$ success probability (1-B). If $A E$ incidence is low, it is assumed to follow Poisson distribution.

$$
\sum_{x=0}^{a-1} \frac{(N \lambda)^{x} e^{N \lambda}}{x !}=\beta
$$
a: number of $A E$ incidences
$\lambda$ : AE incidence rate $(0.13 \%)$
B: probability of not identifying an AE (5\%)
$\mathrm{N}$ : required number of subjects

Based on the above formula, a total of 2,305 subjects were planned for inclusion in this study.

\section{Definition and assessment of AEs and efficacy information}

An AE was defined as any unintended medical event (sign, symptom, or disease) occurring in patients treated with ZNS that did not necessarily have to have a causal relationship to ZNS. Serious AEs included death, life-threatening, hospitalization or prolongation of the hospitalization resulting in persistent impairment, persistent or significant disability or incapacity, and congenital anomaly. Life-threatening $A E$ is defined as a condition where the patient is at the imminent risk of death unless a medical intervention is provided in the opinion of the study physician. To exclude the confounding effect due to frequent hospitalization in the elderly, hospitalization for rest, pre-scheduled before study initiation, and that for routine checkups such as for a physical examination, was excluded from serious AEs. Non-serious AEs were defined as any AE that was not classifiable to serious AEs.

The causal relationship between AE and ZNS treatment was assessed as certain (a clinical event occurring in a plausible time relationship to drug administration, which cannot be explained by other drugs, chemicals, or concurrent disease), probable/likely (a clinical event with a reasonable time sequence to administration of the drug, unlikely to be attributed to other drugs, or concurrent disease), possible (a clinical event with a reasonable time sequence to administration of the drug, but which could also be explained by other drugs, chemicals, or concurrent disease), unlikely (a clinical event with a temporal relationship to drug administration which makes a causal relationship improbable, and in which other drugs, chemical, or underlying disease provide plausible explanations), condition/unclassified (a clinical event about which more data are essential for a proper assessment or the additional data are under examination), and unassessable/unclassifiable (a clinical event which cannot be judged because information is insufficient or contradictory, and which cannot be supplemented or verified).

Patient improvement, tolerability, and global assessment were measured at 12 weeks post-dose in patients who had been administered at least $70 \%$ of ZNS doses over 12 weeks.

\section{Statistical analysis}

All analyses, including demographic data, safety, and efficacy assessments, were carried out as 2-sided tests at a significance level of 


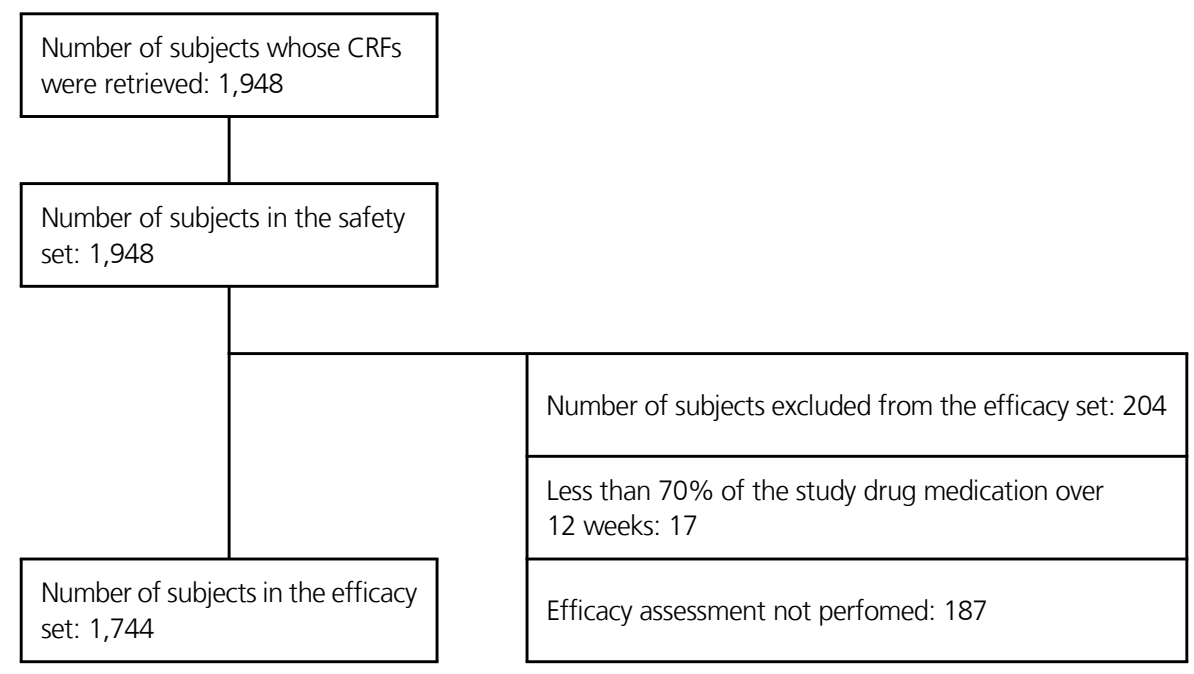

Figure 1. Disposition of patients included in the present study. CRF, clinical record form.

0.05. Mean \pm standard deviation, median, and range (minimum, maximum) were presented as continuous variables, and frequency and proportion were presented as categorical variables. Frequency and proportion were presented as markers of patient improvement, tolerability, and global assessment at Week 12 post-dose of ZNS and the difference by factor was analyzed using Pearson's chi-square test or Fisher's exact test. Since this was an observational study, a missing observation was processed as missing data in the analysis.

\section{Results}

A total of 1,948 patients were included in the study, all of whom were prescribed ZNS at least once and had safety measurements, and thus, were also included in the safety analysis. Among those, 203 patients were excluded from the efficacy analysis due to less than $70 \%$ of the study drug medication (17 patients) over 12 weeks and efficacy assessment not performed (187 patients). The remaining 1,744 patients were analyzed for efficacy (Fig. 1).

\section{Demographic characteristics}

Assessment of the demographic characteristics of patients who participated in this study indicated that $56.21 \%(1,095 / 1,948 \mathrm{pa-}$ tients) were male and $43.79 \%$ (853/1,948 patients) were female. Mean age was $44.2 \pm 20.7$ years. The age distribution of the patients, status of concomitant disease and the number of concomitant AEDs were presented in Table 1.

Mean duration of epilepsy in patients who participated in this study was $7.96 \pm 9.53$ years. Among the 1,948 patients, $69.05 \%$
Table 1. Information on the demographics and concurrent AEDs

\begin{tabular}{|c|c|}
\hline Baseline Information & $\begin{array}{c}\text { Total } \\
(\mathrm{N}=1,948) \\
\mathrm{n}(\%)\end{array}$ \\
\hline \multicolumn{2}{|l|}{ Gender } \\
\hline $\mathrm{N}$ & 1,948 \\
\hline Male & $1,095(56.21)$ \\
\hline Female & $853(43.79)$ \\
\hline \multicolumn{2}{|l|}{ Age (years) } \\
\hline $\mathrm{N}$ & 1,948 \\
\hline Mean \pm SD & $44.21 \pm 20.67$ \\
\hline Median & 45.00 \\
\hline Min, Max & $0.00,93.00$ \\
\hline$<19$ & $240(12.32)$ \\
\hline 19 to $<30$ & $257(13.19)$ \\
\hline 30 to $<40$ & $312(16.02)$ \\
\hline 40 to $<50$ & $342(17.56)$ \\
\hline 50 to $<60$ & $283(14.53)$ \\
\hline 60 to $<70$ & $158(13.24)$ \\
\hline$\geq 70$ & $256(13.14)$ \\
\hline \multicolumn{2}{|l|}{ Children } \\
\hline $\mathrm{N}$ & 1,948 \\
\hline Date of birth to $<24$ months & $16(0.82)$ \\
\hline 24 months to $<12$ years & $122(6.26)$ \\
\hline 12 to 19 years & $102(5.24)$ \\
\hline$\geq 19$ years & $1,708(87.68)$ \\
\hline \multicolumn{2}{|l|}{ Elderly } \\
\hline $\mathrm{N}$ & 1,948 \\
\hline$\geq 65$ years & $387(19.87)$ \\
\hline$<65$ years & $1,561(80.13)$ \\
\hline
\end{tabular}


Table 1. Continued

\begin{tabular}{lc}
\hline Baseline Information & Total \\
& $(\mathrm{N}=1,948)$ \\
$\mathrm{n}(\%)$
\end{tabular}

AED, antiepileptic drug; SD, standard deviation; Min, minumum; Max, maximum; BMl, body mass index

(1,345 patients) had partial seizures, 28.90\% (563 patients) had generalized seizures, and $2.05 \%$ (40 patients) had special syndrome or unclassified.

\section{Status of the study drug administration}

In terms of status of ZNS administration in subjects who participated in this study, the mean daily dose was $186.72 \pm 98.68 \mathrm{mg}$ and the majority of dosing frequency per day was twice daily in $72.74 \%$ (1,417/1,948 patients). 18.48\% (360/1,948 patients) had a dose titration, and mean duration of dose titration and maintenance was
$33.33 \pm 20.60$ days and $59.76 \pm 25.29$ days, respectively.

\section{Safety assessment}

AEs were reported in 3.34\% (65/1,948 patients, 71 events) of the total patients during the study period from the beginning of ZNS administration to the end of observation. The incidence rate of adverse drug reactions (ADRs), serious AEs, and serious ADRs was 2.41\% (47/1,948 patients, 48 events), 0.56\% (11/1,948 patients, 12 events) and $0.05 \%$ (1/1,948 patients, 1 event), respectively. Known ADRs and $A E s$ occurring during the drug-drug interactions were reported in 2.21\% (43/1,948 patients, 44 events) and 0.56\% (11/1,948 patients, 11 events) respectively. 1.89\% (35/1,948 patients, 37 events) experienced AEs leading to permanent discontinuation of the study drug administration.

The most common AEs were 'dizziness' in 0.62\% (12/1,948 patients, 12 events), 'drowsiness' in 0.26\% (5/1,928 patients, 5 events), and 'headache', 'anorexia' and 'general weakness', each in $0.15 \%$ (3/1,948 patients, 3 events). The incidence of serious AEs and serious ADRs collected during the study period was $0.56 \%$ (11/1,948 patients, 12 events) and $0.05 \%$ (1/1,948 patients, 1 event), respectively. The ADRs included two events of 'gastrointestinal disorder', one event of 'interstitial pneumonitis', 'acute respiratory distress syndrome', 'pneumonia', 'diarrhea', hypoalbuminemia', 'sepsis', 'acute renal failure', 'non-small cell lung cancer', 'depression with anxiety', and 'Stevens-Johnson syndrome'. Among the 12 SAE reported, 1 event of 'Stevens-Johnson syndrome' was a serious ADR. This ADR persisted for approximately 4 months from July to November 2011 and was assessed 'possible' in terms of causal relationship to ZNS treatment. As for the action taken, the study drug administration was permanently discontinued and the subject was confirmed to have fully recovered from the ADR. No re-challenge was performed. The incidence rate of unexpected AEs and unexpected ADRs collected during the study period was $0.72 \%$ (14/1,948 patients, 14 events) and 0.21 (4/1,948 patients, four events), respectively. The unexpected AEs included two events of 'unspecified gastrointestinal disorder' in two subjects, and one event of 'epigastric discomfort', 'abdominal discomfort', 'gastritis', 'wooziness', 'cognitive deterioration', 'acute respiratory distress syndrome', 'pneumonia', 'hypoalbuminemia', 'sepsis', 'severe granulocytopenia', 'non-small cell lung cancer', and 'generalized edema', each in one patient. Among the serious AEs and unexpected AEs, there were four deaths due to hypoalbuminemia, acute respiratory distress syndrome, sepsis, and non-small cell lung 
cancer, all of which were assessed 'unlikely' to be related to ZNS treatment.

\section{Efficacy and tolerability assessment}

Patient improvement at Week 12 post-dose of the study drug was assessed as one of the five stages (seizure free, markedly improved (seizure reduction $\geq 50 \%$ ), improved (decrease in seizure frequency 25-50\%), unchanged, and aggravated (increased in seizure frequency)). The majority of the patient improvement assessment was 'seizure free' in $43.29 \%$ (755/1,744 patients), followed by 'markedly improved' in $18.41 \%$ (321/1,744 patients), 'improved' in $21.73 \%$ (378/1,744 patients), 'unchanged' in $16.17 \%$ (282/1,744 patients), and 'aggravated' in $0.40 \%$ (7/1,744 patients). Patients with generalized seizure $(p<0.001)$ and monotherapy $(p<0.001)$ had higher chance of favorable improvement outcome after ZNS treatment.

At week 12 post-dose of the study drug, tolerability to the study drug was assessed as one of the four stages (excellent, good, moderate, bad). Results from the tolerability assessment indicated that the majority was 'good' in 58.03\% (1,012/1,744 patients), followed by 'excellent' in 26.43 (461/1,744 patients), 'moderate' in 15.08\% (263/1,744 patients), and 'bad' in 0.46\% (8/1,744 patients).

At week 12 post-dose of the study drug, the physician and the patient each measured global assessment as one of the three stages (improved, unchanged, and aggravated). Results from the global assessment measured by the physician indicated that the majority was 'improved' in $73.58 \%(1,281 / 1,741$ patients), followed by 'unchanged' in 26.08\% (454/1,741 patients), and aggravated in 0.34\% $(6 / 1,741$ patients). The majority of global assessment measured by the patient was 'improved' in $70.96 \%(1,229 / 1,732$ patients), followed by 'unchanged' in $28.41 \%$ (492/1,732 patients), and 'aggravated' in $0.64 \%$ (11/1,732 patients).

\section{Discussion}

A drug's safety and efficacy must be demonstrated in a series of clinical trials conducted prior to the approval of the drug. Randomized controlled trials are considered to be the most rigorous approach to determining cause-and-effect relationship between the drug and AEs. The controlled nature of such trials, however, calls for a limited number of patients who may not always be representative of the population of all potential users of the drug and a relatively short observation period, making it difficult to detect ADRs that are rare or with a long latency. ${ }^{9}$ Post-marketing drug surveillance refers to the monitoring of drugs once they reach the market after clinical trials. It evaluates drugs taken by individuals under a wide range of circumstances over an extended period of time. Such surveillance is much more likely to detect previously unrecognized positive or negative effects that may be associated with a drug. The majority of post-marketing surveillance concern ADRs monitoring and evaluation. ${ }^{10}$

In this study, a total of 1,948 patients who used ZNS provided safety information and were assessed for safety. Among those, 1,477 patients were assessed for efficacy. In the study planning phase, the sample size was determined by using the incidence rate of renal stone which is a rare AE. To identify one incidence at a $95 \%$ probability, it was planned to recruit a total 2,305 patients. However, as the information was collected from 1,948 patients in reality, the probability of indentifying one AE incidence of renal stone declined to $92 \%$.

During the study, 71 AEs were reported in 65 out of 1,948 patients, resulting in an AE incidence rate of $3.34 \%$. The incidence of AE was lower than previously documented in randomized or longterm observational studies. ${ }^{11-13}$ The frequency at which adverse events of AEDs are reported in a given population is dependent on the method of assessment, and every method has limitation. Reliance on unstructured interviews or spontaneous reporting underestimates the burden of AED toxic effects, whereas use of screening measures, such as questionnaires or checklists, can result in overestimation. ${ }^{14}$ In the present study, the mean daily dose used $(186.72 \pm 98.68 \mathrm{mg})$ was lower than the usual maintenance dose (300-400 mg/day), and this low dosage may associated with the low incidence of AEs. More importantly, the primary objective of this post-marketing surveillance study was to investigate and collect the safety information of ZNS in the clinical setting, focusing on the unexpected or serious $A E s$, so it is possible that the physicians neglected the occurrence of mild or commonly observed AEs during the ZNS treatment. Similarly with our results, the incidence of lamotrigine-related skin rash was much lower in postmarketing surveillance $(2.09 \%)$ compared with those of the prospective clinical trials (9.98\%) and retrospective trials $(7.19 \%){ }^{15}$ There was one serious ADR in one patient of which causal relationship to ZNS cannot be ruled out. Specifically, this was 'Stevens-Johnson syndrome'. This event was assessed as 'possible' for causal relationship to ZNS and was reported to have fully recovered after the study drug as permanently discontinued. In general, Stevens-Johnson syndrome is a serious but rare AE in ZNS treatment, ${ }^{16}$ and a recent study suggested a genetic predisposition in the development of StevensJohnson syndrome in Japanese population. ${ }^{17}$ Special caution was ex- 
ercised to monitor possible AEs of renal stone as this was noted in the precautions for use in the approval in Korea based on the results of previous study of ZNS. However, no renal stone as an AE occurred in this study and in previous studies in Korea. ${ }^{1,8}$

In terms of efficacy of ZNS, results from the evaluation of patient improvement, tolerability, and global assessment measured at Week 12 post-dose indicated that the study drug had good tolerability and efficacy in most patients. In the clinical improvement assessment results, the majority was 'seizure free' in $43.29 \%$ followed by 'markedly improved' in $18.41 \%$, which indicated that at least half of the subject showed at least $50 \%$ improvement in seizures. In terms of tolerability, the majority was 'good' in $58.03 \%$, followed by 'excellent' in $26.43 \%$, which indicated that at least $80 \%$ of patients had good tolerability. In terms of physician's and patient's global assessment, the majority was 'improved' in $73.58 \%$ and $70.96 \%$, respectively, which indicated that the symptoms maintained or improved in at least $99 \%$ of the patients after ZNS treatment. The efficacy and tolerability of ZNS as adjuvant therapy in adults with refractory partial epilepsy have been demonstrated in several randomized controlled trials. ${ }^{18}$ However, the clinical application of these trial results is limited because of the short-term duration of study, strict control of concomitant AEDs, and recruitment of patients who satisfy strict inclusion criteria. $^{19}$ Several long-term observational studies have shown the clinical usefulness of ZNS in adult patients with partial epilepsy. ${ }^{4,20,21}$ But, the majority of the enrolled patients used ZNS as an add-on drug for partial seizures because ZNS is usually restricted for the adjunctive treatment of partial seizures in the USA and Europe. $^{18}$ The present study shows that efficacy of ZNS treatment was more favorable in patients with monotherapy and generalized seizure, which is consistent with recent long-term observation study with epilepsy patients in Korea. ${ }^{13}$

In conclusion, as a result of administration of ZNS to patients with epilepsy, there were four unexpected ADRs, all of which, however, were non-serious ADRs. Based on the approval in Korea and currently available drug information, no other notable matters expected to affect the safety of ZNS were identified. In addition, ZNS is considered to be well tolerated and to significantly improve symptoms based on the physician's and patient's global assessment. Therefore, administration ZNS to patients with epilepsy including partial and generalized seizures for therapeutic purpose is considered to be safe and effective.

\section{References}

1. Park SP, Kim SY, Hwang YH, Lee HW, Suh CK, Kwon SH. Long-term efficacy and safety of zonisamide monotherapy in epilepsy patients. J Clin Neurol 2007;3:175-80.

2. Zaccara G, Specchio LM. Long-term safety and effectiveness of zonisamide in the treatment of epilepsy: A review of the literature. Neuropsychiatr Dis Treat 2009;5:249-59.

3. Brodie MJ, Ben-Menachem E, Chouette I, Giorgi L. Zonisamide: Its pharmacology, efficacy and safety in clinical trials. Acta Neurol Scand Supp/ 2012;(194):19-28.

4. Chung S, Wang N, Hank N. Comparative retention rates and long-term tolerability of new antiepileptic drugs. Seizure 2007;16:296-304.

5. Oommen KJ, Mathews S. Zonisamide: A new antiepileptic drug. Clin Neuropharmacol 1999;22:192-200.

6. Brodie MJ, Duncan R, Vespignani H, Solyom A, Bitenskyy V, Lucas C. Dose-dependent safety and efficacy of zonisamide: A randomized, double-blind, placebo-controlled study in patients with refractory partial seizures. Epilepsia 2005;46:31-41.

7. Ohtahara S, Yamatogi Y. Safety of zonisamide therapy: Prospective follow-up survey. Seizure 2004;13 Suppl 1:S50-5

8. Korean Zonisamide Study Group. Double-blind, randomized, comparative clinical trial of zonisamide and carbamazepine as initial monotherapy in newly diagnosed epilepsy. J Korean Epilepsy Soc 1999;3:50-7.

9. Coloma PM, Trifiro G, Patadia V, Sturkenboom M. Postmarketing safety surveillance: Where does signal detection using electronic healthcare records fit into the big picture? Drug Saf 2013;36:183-97.

10. Vlahovic-Palcevski V, Mentzer D. Postmarketing surveillance. Handb Exp Pharmacol 2011;205:339-51.

11. Yamauchi T, Aikawa H. Efficacy of zonisamide: Our experience. Seizure 2004;13 Suppl 1:S41-4.

12. Baulac M, Brodie MJ, Patten A, Segieth J, Giorgi L. Efficacy and tolerability of zonisamide versus controlled-release carbamazepine for newly diagnosed partial epilepsy: A phase III, randomised, double-blind, non-inferiority trial. Lancet Neurol 2012;11:579-88.

13. Kim DW, Choi K, Moon HS, Oh J. Long-term retention rate of zonisamide in patients with epilepsy: An observational study. Clin Neuropharmacol 2014;37:133-5.

14. Perucca P, Gilliam FG. Adverse effects of antiepileptic drugs. Lancet Neurol 2012;11:792-802.

15. Wang $X Q$, Xiong J, Xu WH, et al. Risk of a lamotrigine-related skin rash: Current meta-analysis and postmarketing cohort analysis. Seizure. 2015;25:52-61.

16. Baulac M, Patten A, Giorgi L. Long-term safety and efficacy of zonisamide versus carbamazepine monotherapy for treatment of partial seizures in adults with newly diagnosed epilepsy: Results of a phase III, randomized, double-blind study. Epilepsia 2014;55:1534-43.

17. Kaniwa N, Sugiyama E, Saito Y, Kurose K, Maekawa K, Hasegawa R, et al. Specific HLA types are associated with antiepileptic drug-induced 
Stevens-Johnson syndrome and toxic epidermal necrolysis in Japanese subjects. Pharmacogenomics 2013;14:1821-31.

18. Dupont S, Stefan H. Zonisamide in clinical practice. Acta Neurol Scand Supp/ 2012;(194):29-35.

19. Walker MC, Sander JW. Topiramate: A new antiepileptic drug for refractory epilepsy. Seizure 1996;5:199-203.
20. Wroe SJ, Yeates AB, Marshall A. Long-term safety and efficacy of zonisamide in patients with refractory partial-onset epilepsy. Acta Neurol Scand 2008;118:87-93.

21. Catarino CB, Bartolini E, Bell GS, Yuen AW, Duncan JS, Sander JW. The long-term retention of zonisamide in a large cohort of people with epilepsy at a tertiary referral centre. Epilepsy Res 2011;96:39-44. 